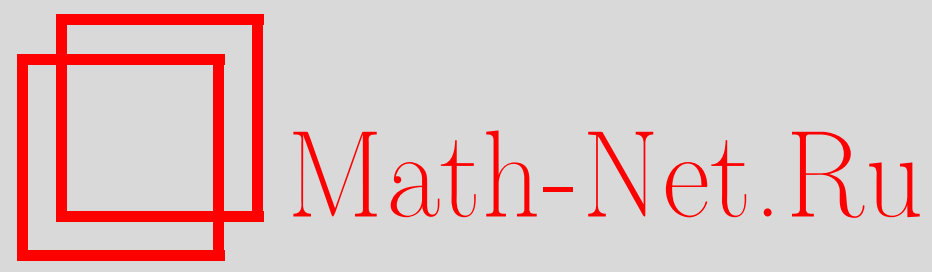

М. Г. Шур, Квазифеллеровские расширения марковских цепей и существование двойственных цепей, Матем. заметки, 2001, том 69, выпуск 1, 133-143

DOI: https://doi.org/10.4213/mzm490

Использование Общероссийского математического портала Math-Net.Ru подразумевает, что вы прочитали и согласны с пользовательским соглашением http://www.mathnet.ru/rus/agreement

Параметры загрузки:

IP : 52.6 .47 .48

26 апреля 2023 г., 18:30:38

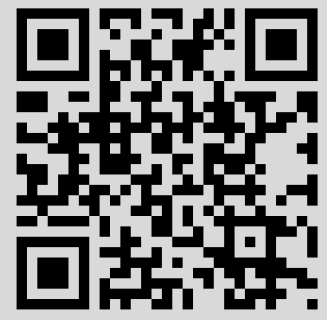




\title{
КВАЗИФЕЛЛЕРОВСКИЕ РАСШИРЕНИЯ МАРКОВСКИХ ЦЕПЕЙ И СУЩЕСТВОВАНИЕ ДВОЙСТВЕННЫХ ЦЕПЕЙ
}

М.Г. Шур

\begin{abstract}
Предложен исправленный и детализированный вариант теоремы автора о существовании феллеровских расширений марковских цепей, для чего использовано более широкое понятие квазифеллеровского расширения. Из этого резултата выводится существование марковских цепей, двойственных цепям с борелевскими пространствами состояний. Наиболее подробно изучены расширения неприводимых в смысле Ори цепей. $\mathrm{K}$ примеру, показано, что для таких цепей квазифеллеровские расширения можно выбрать возвратньми или лиувиллевьми, коль скоро таковыми являются исходные це-

Библиография: 11 названий.
\end{abstract} пи.

1. Введение. В теореме 1 работы [1] обсуждался вопрос о построении феллеровских расширений цепей Маркова, заданных в сепарабельных измеримых пространствах состояний. Доказательство указанной теоремы, будучи вполне корректньп в важном случае борелевских пространств (определение см. в п. 2), в общем случае неудовлетворительно. Это побудило нас вернуться к той же проблематике, одновременно несколько изменив определение расширения цепи и включив в рассмотрение квазифеллеровские расширения.

В теореме 1 предлагаемой работы с помощью теоремы Стоуна-Вейерштрасса установлено существование таких расширений, что позволило легко доказать существование цепей, двойственных, в частности, цепям Маркова с борелевскими пространствами состояний (см. теорему 2 и замечание 3). Заключительная часть работы $($ пा. 5, 6) отведена расширениям неприводимых цепей. Несколько ограничив в этом случае свободу выбора расширений (см. условие (III)), мы обнаруживаем в теореме 4, что соответствуюшие расширения сохраняют неприводимость, а их параметры сходимости совпадают с таковыми для исходных цепей. Кроме того, соответствуюшие расширения наследуют свойства типа возвратности или лиувиллевости (теоремы 5 и 6). Попутно обсуждается связь между классами минорантных множеств в исходных и компактных множествах в расширенных пространствах состояний (см. теорему 3, прообразом которой служит теорема 2 из [1]).

В одной из ближайших работ автор намерен показать, как указанные результаты помогают получить новые предельные теоремы для отношений.

Работа выполнена при поддержке Российского фонда фундаментальных исследований, грант № 98-01-529. 
2. Основные обозначения и терминология. Остановимся на обозначениях и терминологии основной части работы (пп. 1-4) (специфика случая неприводимых цепей отражена в добавлении (п. 6)).

На протяжении работы фиксируется измеримое пространство $(E, \mathscr{B})$, удовлетворяющее условию

(I) $\sigma$-алгебра $\mathscr{B}$ является сепарабельной и содержит все одноточечные множества в $E$.

Важным примером такого пространства служит борелевское (в иной терминологиилузинское) пространство, являющееся, по определению, изоморфным $\left(E_{0}, \mathscr{B}_{0}\right)$, где $E_{0}$ - борелевское множество в некотором полном сепарабельном метрическом пространстве $M$, а $\mathscr{B}_{0}$ - семейство всех борелевских подмножеств этого множества. Символ $\mathscr{B}$ будет обозначать также совокупность всех измеримых функций $f: E \rightarrow \mathbb{R} \cup\{+\infty\}$, а под $\mathscr{B}+($ соответственно $b \mathscr{B})$ будет пониматься ее часть, образованная неотрицательными (ограниченными) элементами. Иногда будет удобным обозначение $\mu(f)=\int f d \mu$, если указанный интеграл имеет смысл, где $f$ - функция на $E$, а $\mu$-мера на $\mathscr{B}$. Аналогичные соглашения действуют и в случае других измеримых пространств.

В $(E, \mathscr{B})$ задается (однородная) цепь Маркова $X=\left\{X_{n} ; n \geqslant 0\right\}$ с субмарковской переходной вероятностью $p(x, A), x \in E, A \in \mathscr{B}$, и переходньм оператором $P$, действующим либо в $\mathscr{B}_{+}$, либо в $b \mathscr{B}$ (см. [2], [3]). Точку $x_{0} \in E$ назовем әфемерной, если $p\left(x_{0}, E\right)=0$ и $p\left(\cdot,\left\{x_{0}\right\}\right) \equiv 0$. При ее наличии разумно $E$ заменить на $E^{\prime}=E \backslash\left\{x_{0}\right\}, \mathscr{B}-$ на след $\mathscr{B}^{\prime} \sigma$-алгебры $\mathscr{B}$ в $E^{\prime}$ и вместо $p(\cdot, \cdot)$ рассмотреть соответствующее сужение на $\left(E^{\prime}, \mathscr{B}^{\prime}\right)$. Последнему отвечает цеп Маркова на $\left(E^{\prime}, \mathscr{B}^{\prime}\right)$, называемая частью $X$ на $E^{\prime}$.

Переходная вероятность цепи $X$ за $n \geqslant 1$ шагов и порождаемый ею переходньй оператор обозначаются $p_{n}(\cdot, \cdot)$ и $P^{n}$ соответственно (таким образом, $p_{1} \equiv p$ ). Считая впредь $\alpha \in(0,1)$ фиксированным, определим для $f \in b \mathscr{B}$ (а также для $f \in \mathscr{B}_{+}$) оператор $G^{\alpha}$ :

$$
G^{\alpha} f=f+\sum_{n \geqslant 1} \alpha^{n} P^{n} f .
$$

Правая часть (1) с $\alpha$, замененным на 1 , определяет функцию $G f$ для $f \in \mathscr{B}_{+}$. Если $G f \equiv \infty$ для всех $f \in \mathscr{B}_{+}$, цепь $X$ назьвают возвратной. Она же $\nu$-неприводима $[2],[3]$ $(\nu-\sigma$-конечная мера на $\mathscr{B})$, если $P G\left(1_{A}\right)>0$ в $E$ для любого $A$ с $\nu(A)>0$, где $1_{A}$ - индикатор множества $A$ (при этом $\nu$ именуется мерой, определяющей неприводимость). Цепь $X$ по определению лиувиллева (соответственно $\nu$-лиувиллева, где снова $\nu-\sigma$-конечная мера на $E$ ), если существует ненулевая $\sigma$-конечная инвариантная для $X$ (и одновременно абсолютно непрерьвная относительно $\nu$ ) мера, которой пропорциональны остальные меры указанного вида. Наконец, допустив, что $E$ является локально компактным пространством, а $\mathscr{B}$ - совокупностью его борелевских множеств, назовем цепь $X$ феллеровской (квазифеллеровской в случае некомпактного $E$ ), если $P$ отображает в себя совокупность $C(E)$ всех ограниченных непрерьвных в $E$ функций (соответственно всех $f \in C(E)$, стремящихся к 0 на бесконечности). Квазифеллеровская цепь $X$ является феллеровской тогда и только тогда, когда функция $P 1$ непрерьвна.

Пусть имеется еще одна цепь Маркова $\bar{X}$, реализованная на измеримом пространстве состояний $(\bar{E}, \overline{\mathscr{B}})$, с переходной вероятностью $\bar{p}(\cdot, \cdot)$, и пусть существует измеримая инъекция $\varphi:(E, \mathscr{B}) \rightarrow(\bar{E}, \overline{\mathscr{B}})$. Назовем $\bar{X}$ расширением цепи $X$, отвечающим $\varphi$, если $\varphi(E)$ плотно в $\bar{E}$ и при любом $x \in E$ мера $\bar{p}(\varphi(x), \cdot)$ является образом меры $p(x, \cdot)$ 
(т.е. $\bar{p}(\varphi(x), A)=p\left(x, \varphi^{-1}(A)\right)$ для $x \in E$ и $\left.A \in \overline{\mathscr{B}}\right)$. Это определение равносильно использованному в $[1]$, если пространства $(E, \mathscr{B})$ и $(\bar{E}, \overline{\mathscr{B}})$ являются борелевскими.

3. Существование квазифеллеровских расширений. Конструкции феллеровских расширений цепей Маркова, основанные на идеях Стоуна-Чеха или теории банаховых алгебр, обычно приводят к чрезмерному разрастанию исходного пространства состояний [4]. В [1] была предложена более экономная конструкция, позволившая при условии (I) строить феллеровские расширения в метрических компактах. Возвращаясь к ней, мы приводим в этом пункте теорему 1 - исправленньй и детализированный вариант теоремы 1 из [1], используя при доказательстве ту же идею $\Phi$. Найта [5], что и прежде.

Будем говорить, что функции $h_{n} \in b \mathscr{B}, n \geqslant 1$, образуют допустимый набор, если они, во-первых, разделяют точки в $E$ (т.е. если для любых $x, y \in E(x \neq y)$ найдется $n \geqslant 1$, при котором $\left.h_{n}(x) \neq h_{n}(y)\right)$, и во-вторьх, порождают $\mathscr{B}$. Например, индикаторы $u_{n}=1_{A_{n}}$ множеств $A_{n}, n \geqslant 1$, порождаюших $\mathscr{B}$ (см. условие (I)), образуют требуемьй набор. В самом деле, пусть $u_{n}(x)=u_{n}(y)$ при $n \geqslant 1$ и некоторьх фиксированных $x, y \in E(x \neq y)$. Ясно, что $\sigma$-алгебра $\mathscr{B}_{0} \subset \mathscr{B}$, составленная из всех $A \in \mathscr{B}$, содержащих или нет сразу обе выбранные точки, включает в себя любое множество вида $\left\{z \in E: u_{n}(z)<d\right\}, n \geqslant 1, d \in \mathbb{R}$, а потому функции $u_{n}$ измеримы относительно $\mathscr{B}_{0}$. Так как они порождают $\mathscr{B}$, то $\mathscr{B}_{0}=\mathscr{B}$, вопреки тому, что одноточечное множество $\{x\}$ входит в $\mathscr{B}$, но не в $\mathscr{B}_{0}$. Это противоречие и свидетельствует о допустимости указанного набора.

Фиксируем до конца пункта допустимьй набор $H_{0}=\left\{h_{n} ; n \geqslant 1\right\}$.

Лемма 1. Существует алгебра функиий $\mathfrak{A} \subset$ bЯ̈, инвариантная относительно $P$, разделяющая точки в $E$ и содержащая $H_{0}$ и некоторое не более чем счетное плотное в ней (в топологии равномерной сходимости) подмножсество. Более того, А мохнн считать наименьшей среди всех алгебр в бЯ̈, обладающих указанными свойствами.

ДокАЗАТЕЛЬСТво. Для каждого семейства $H \subset b \mathscr{B}$ образуем наименьшую алгебру (над полем $\mathbb{R}) \mathfrak{A} H \subset b \mathscr{B}$, содержащую $H$. Алгебры $H_{n}, n \geqslant 1$, определим последовательно, полагая $H_{n}=\mathfrak{A}\left(H_{n-1} \cup P H_{n-1}\right)$, где $P H_{n-1}-$ образ $H_{n-1}$ при действии $P$. Легко проверяется (см. [1, с. 498]), что алгебра $\mathfrak{A}=\bigcup_{n \geqslant 0} H_{n}$ является искомой.

Для дальнейшего существенно, что каждая функция вида $G^{\alpha} h_{n}, n \geqslant 1$, лежит в равномерном замыкании алгебры $\mathfrak{A}$.

Tеорема 1. Существуют метрический компакт $\bar{E} \supset$ Е и измеримое отображение $\varphi:(E, \mathscr{B}) \rightarrow(\bar{E}, \overline{\mathscr{B}})$, где $\overline{\mathscr{B}}-$ борелевская $\sigma$-алгебра в $\bar{E}$, со следующими свойствами:

а) множество $E=\varphi(E)$ плотно в $\bar{E}$ и любая функиия $f$, входящая в равномерное замыкание алгебры $\mathfrak{A}$ (см. лемму 1), допускает единственное продолэение $\bar{f} \in C(\bar{E}) ;$ при этом в $\bar{E}$ имеется не более одной такой точкu $\Delta$, что $\overline{G^{\alpha} h_{n}}(\Delta)=0$ при всех $n \geqslant 1$;

б) в $(\bar{E}, \overline{\mathscr{B}})$ задано расиирение $\bar{X}$ исходной цепи Маркова $X$, причем справедлива альтернатива: либо указанная точка $\Delta$ не существует и тогда $\bar{X}$ является феллеровской цепью Маркова, либо әта точка существует и 
тогда она служит әфемерным состоянием для $\bar{X}$, а часть $\widehat{X}$ иепи $\bar{X}$ на мнохестве $\widehat{E}=\bar{E} \backslash\{\Delta\}$ является квазифеллеровской иепью.

ДокАЗАТЕЛЬСтво. Пусть последовательность $\left\{g_{n} \in \mathfrak{A} ; n \geqslant 1\right\}$ образует плотное в $\mathfrak{A}$ множество (см. лемму 1$)$. Наделив $E$ метрикой $\rho(x, y)=\sum_{n \geqslant 1} 2^{-n} w\left[g_{n}(x)-g_{n}(y)\right]$, $x, y \in E$, где положено $w[t]=|t| /(1+|t|)$ для $t \in \mathbb{R}$, рассмотрим компакт $\bar{E}$, являюшийся пополнением полученного метрического пространства. Как и в [1], каждая из функций $f \in \mathfrak{A}$ (а стало быть, и каждая функция из равномерного замыкания алгебры $\mathfrak{A})$, будучи равномерно непрерьвной в метрике $\rho$, обладает единственным продолжением $\bar{f} \in C(\bar{E})$. В частности, определено продолжение $\bar{g}_{n} \in C(\bar{E})$ для $g_{n}$.

Семейство $\left\{\bar{g}_{n}\right\}$ разделяет точки в $\bar{E}$ : если $\bar{g}_{m}(x)=\bar{g}_{m}(y)$ при всех $m \geqslant 1$ и некоторых $x, y \in \bar{E}$, а $x_{n}, y_{n} \in E$ таковы, что $x_{n} \rightarrow x, y_{n} \rightarrow y$ при $n \rightarrow \infty$, то $g_{m}\left(x_{n}\right)-g_{m}\left(y_{n}\right)$ $\rightarrow 0$ при любом $m \geqslant 1$, вследствие чего $x=y$. Таким образом, семейство $\overline{\mathfrak{A}}=\{\bar{f}: f \in \mathfrak{A}\}$ является алгеброй непрерьвных функций в $\bar{E}$, разделяющей точки, и по теореме Стоуна-Вейерштрасса [6] равномерноезамыкание этой алгебры совпадает либо с $C(\bar{E})$ (случай I), либо с $C_{0}(\bar{E})=\left\{f \in C(\bar{E}): f\left(x_{0}\right)=0\right\}$, где $x_{0}$-некоторая точка в $\bar{E}$ (случай II).

Пусть $\mathscr{B}_{1}-$ след $\sigma$-алгебры $\overline{\mathscr{B}}$ на $E$ (таким образом, $\mathscr{B}_{1}$ состоит из всевозможных пересечений $A \cap E, A \in \overline{\mathscr{B}})$. Докажем, что

$$
\mathscr{B}_{1}=\mathscr{B}
$$

Сужение на $E$ любой функции из $\overline{\mathfrak{A}} \mathscr{B}$-измеримо, и потому $\mathscr{B}$-измеримы сужения на $E$ всех $f \in C(\bar{E})$ (в случае II этот факт вытекает из его справедливости для $f \in C_{0}(\bar{E})$ и для $f$, сохраняющих постоянство). Ввиду известной версии теоремы о монотонньх классах (см. [7, теорема 1.21]) то же относится и к сужениям любых $f \in b \overline{\mathscr{B}}$, так что $\mathscr{B}_{1} \subset \mathscr{B}$. Обратное же включение очевидно, так как функции $h_{n}$, будучи $\mathscr{B}_{1}$-измеримыми, порождают $\mathscr{B}$. Итак, (2) доказано, и тем самьм установлена измеримость вложения $\varphi: E \rightarrow \bar{E}$, определяемого равенством $\varphi(x)=x, x \in E$.

Приступим к определению подходящей переходной вероятности в $(\bar{E}, \overline{\mathscr{B}})$. Пусть $f_{E}$ обозначает сужение на $E$ функции $f \in \overline{\mathscr{B}}$. Если $f \in \overline{\mathfrak{A}}$, то $P\left(f_{E}\right) \in \mathfrak{A}$ в силу инвариантности $\mathfrak{A}$ относительно $P$ и, следовательно, существует единственное продолжение $\overline{P\left(f_{E}\right)}$ класса $C(\bar{E})$ в случае I или класса $C_{0}(\bar{E})$ в случае II, когда $f$ берется из $C(\bar{E})$ или $C_{0}(\bar{E})$ соответственно. Кроме того, $\overline{P\left(f_{E}\right)}(x)$ при фиксированном $x \in \bar{E}$ является ограниченным линейным функционалом в $C(\bar{E})$ (случай I) или $C_{0}(\bar{E})$ (случай II). Следовательно, в случае I для каждого $x \in \bar{E}$ существует единственная мера $\bar{p}(x, \cdot)$ на $\overline{\mathscr{B}}$ такая, что

$$
\overline{P\left(f_{E}\right)}(x)=\int f(y) \bar{p}(x, d y)
$$

для $f \in C(\bar{E})$ и $x \in \bar{E}$ (см. [8, $\S 56$, теорема 4]). Применив в случае II эту же теорему к $\widehat{E}=\bar{E} \backslash\left\{x_{0}\right\}$, получим для $x \in \bar{E}$ аналогичную меру $\bar{p}(x, \cdot)$ на $\overline{\mathscr{B}}$, сосредоточенную в $\widehat{E}$, и положим $\bar{p}\left(x_{0}, \cdot\right)=0$. Заметим, что из непрерывности функций $\overline{P\left(f_{E}\right)}$ для $f$ из соответствующего класса $C(\bar{E})$ или $C_{0}(\bar{E})$ и из упоминавшейся версии теоремы о монотонных классах следует $\overline{\mathscr{B}}$-измеримость функций вида $\bar{p}(\cdot, A), A \in \overline{\mathscr{B}}$. 
Переходной вероятности $\bar{p}$ отвечает некоторая цеп Маркова $\bar{X}$ на $(\bar{E}, \overline{\mathscr{B}})$ с переходным оператором $\bar{P}$, являющаяся расширением исходной цепи. Действительно, с учетом (3) для $x \in E$ и $f \in C(\bar{E})$ находим в случае I, что

$$
\begin{aligned}
\bar{P} f(x) & =\int f(y) \bar{p}(x, d y)=\overline{P\left(f_{E}\right)}(x)=\int f_{E}(y) p(x, d y) \\
& =\int f(\varphi(y)) p(x, d y)=\int f(y) \tilde{p}(x, d y)
\end{aligned}
$$

где $\tilde{p}(x, \cdot)$ - образ меры $p(x, \cdot)$ при введенном выше отображении $\varphi$. Равенство первого и последнего интегралов в (4) остается в силе для всех $f \in b \overline{\mathscr{B}}$ и, следовательно, $\bar{p}(x, \cdot) \equiv \tilde{p}(x, \cdot)$. Оно же сохраняет силу и в случае II, но при дополнительном условии, что $f=0$ вне некоторой окрестности точки $x_{0}$. Это условие легко ослабляется до требования, чтобы $f\left(x_{0}\right)=0$, но и новое требование излишне, так как $\bar{p}\left(x,\left\{x_{0}\right\}\right)=0$. Итак, опять $\bar{p}(x, \cdot) \equiv \tilde{p}(x, \cdot)$ для $x \in E$ и $\bar{X}$ является расширением цепи $X$.

Теперь заметим, что лемма 1.1 из [1], по существу, утверждает, что если существует точка $\Delta \in \bar{E}$, в которой $\overline{G^{\alpha} h_{n}}(\Delta)=0, n \geqslant 1$, то $\bar{f}(\Delta)=0$ для всех $f \in \mathfrak{A}$ (следует лиш учесть, что теперь (1.2) из [1] вьполняется с $d=0)$. Иными словами, такая точка существует лиш в случае II и совпадает с $x_{0}$. Поэтому утверждение теоремы о феллеровости или квазифеллеровости $\bar{X}$ или $\widehat{X}$ вытекает из обсуждения, предшествующего (3).

ЗАмЕчаниЕ 1 . В процессе доказательства теоремы 1 было установлено, что соответствующая цепь $\bar{X}$ обладает свойствами: а) $\sigma$-алгебра $\mathscr{B}$ совпадает со следом $\overline{\mathscr{B}}$ на $E$ (см. (2)), и б) для всех $f \in b \overline{\mathscr{B}}$ и $x \in E$

$$
\bar{P} f(x)=P\left(f_{E}\right)(x),
$$

где снова $f_{E}$ - сужение $f$ на $E$. Аналогичными свойствами обладает и цепь $\widehat{X}$.

ЗАмЕчаниЕ 2. Если исходная цепь $X$ не обрывается (иньми словами, имеет марковскую переходную вероятность), то за счет добавления к допустимому набору $\left\{h_{n}\right\}$ функции $h_{0} \equiv 1$ можно гарантировать отсутствие обрыва $\bar{X}$ и тем самым феллеровское свойство для $\bar{X}$, поскольку в этом случае $\overline{\mathfrak{A}}$ и $C(\bar{E})$ содержат константу 1 и $P 1 \equiv 1$. сожалению, упомянутое добавление функции $h_{0}$ не всегда приемлемо (см. далее условие (III)).

В дальнейшем за символами $f_{E}, \bar{X}, \bar{E}$ и т.п. будет сохранен их смысл, указанный в теореме 1 и замечании 1 (в частности, будет предполагаться, что $\bar{X}$ обладает свойствами а) и б) из замечания 1). Cмысл же обозначений $\widehat{E}$ и $\widehat{X}$ будет расширен: как и прежде, продолжаем считать $\widehat{E}=\bar{E} \backslash\{\Delta\}$ и за $\widehat{X}$ принимаем часть $\bar{X}$ на $\widehat{E}$, если существует точка $\Delta \in \bar{E}$ (см. теорему 1 ), и полагаем $\widehat{E}=\bar{E}$ и $\widehat{X}=\bar{X}$ в противном случае. Обозначения $\widehat{\mathscr{B}}, \widehat{P}$ и т.п. введем по аналогии с $\overline{\mathscr{B}}, \bar{P}$ и т.п. Наконец, естественные вложения $\varphi: E \rightarrow \bar{E}$ или $\varphi: E \rightarrow \widehat{E}$ определим одним и тем же равенством $\varphi(x)=x$, $x \in E$ (в доказательстве теоремы 1 участвовало первое из них).

4. Существование двойственных цепей. Понятие двойственной (или обращенной) цепи играет существенную роль в теории счетных цепей Маркова [9]. Это же понятие используется и в теории цепей Маркова с измеримыми пространствами состояний, 
однако в общем случае в теорему существования двойственных цепей приходится включать требование регулярности меры, определяющей двойственность, относительно того или иного компактного класса множеств [10].

В этом пункте показано (см. теорему 2 , а также замечание 3 ), что теорема 1 позволяет избавиться от подобных предположений, если вьполнено достаточно общее условие

(II) измеримое пространство $($ Е, Я) является борелевским.

Лемма 2. Условие (II) реализуется тогда и только тогда, когда каждое множество из $\mathscr{B}$ является борелевским в пространстве $\widehat{E}$, m.е. когда $\mathscr{B} \subset \widehat{\mathscr{B}}$ (nо поводу символов $\widehat{E}, \widehat{B}$ и т.n. см. конеи п. 3).

ДокАЗАТЕЛЬСтво. Из условия $\mathscr{B} \subset \widehat{\mathscr{B}}$ вытекает (II), причем в качестве метрического пространства $M$ из определения борелевского пространства (см. п. 2) можно взять $E$. Пусть теперь отображение $\psi: E_{0} \rightarrow E$ устанавливает изоморфизм между $\left(E_{0}, \mathscr{B}_{0}\right)$ и $(E, \mathscr{B})$, где $\left(E_{0}, \mathscr{B}_{0}\right)$ - измеримое пространство из того же определения. Рассматривая $\psi$ как инъекцию $E_{0}$ и $\widehat{E}$, получим измеримое отображение $\left(E_{0}, \mathscr{B}_{0}\right)$ в $(\widehat{E}, \widehat{\mathscr{B}})$, поскольку $\psi^{-1}(A)=\psi^{-1}(A \cap E) \in \mathscr{B}_{0}$ для $A \in \widehat{\mathscr{B}}$. Отсюда следует (см. теорему Суслина-Лузина в [6], а также [10]), что каждое множество вида $\psi(B), B \in \mathscr{B}_{0}$, является борелевским в $\widehat{E}$, а именно из множеств такого вида и состоит $\mathscr{B}$.

ТЕОРема 2. Пусть выполнено условие (II), и пусть $\mu-\sigma$-конечная субинвариантная мера для цепи $X$. Тогда в $(E, \mathscr{B})$ существует иепь Маркова, двойственная $\kappa X$ относительно $\mu$ (т.е. чепь с переходным оператором $P^{*}$, для которого $\mu(g P f)=\mu\left(f P^{*} g\right)$ nри всех $\left.f, g \in \mathscr{B}_{+}\right)$.

ДоКАЗАТЕЛЬСТВО. Тривиальньй случай $\mu \equiv 0$ исключим из рассмотрения. Пусть имеются допустимьй набор функций $\left\{h_{n}\right\}$ и множества $E_{1} \subset E_{2} \ldots$ с $\bigcup_{n} E_{n}=E$ и $\mu\left(E_{n}\right)<\infty, n \geqslant 1$. Тогда элементы нового допустимого набора, составленного из всевозможных произведений $h_{n} 1_{E_{m}}, m, n \geqslant 1$, суммируемы по $\mu$. Поэтому, не ограничивая общности, сразу предположим таковыми все элементы исходного набора.

Пусть мера $\hat{\mu}$ на $\widehat{\mathscr{B}}$ служит образом $\mu$ при естественном вложении $E$ в $\widehat{E}$. Непрерывное продолжение $g_{n}=\overline{G^{\alpha} h_{n}}$ функции $G^{\alpha} h_{n}, n \geqslant 1$, суммируемо по $\hat{\mu}$, так как (см., например, [7, теорема 2.12]) $\hat{\mu}\left(g_{n}\right)$ совпадает с

$$
\mu\left(G^{\alpha} h_{n}\right)=\sum_{m \geqslant 0} \alpha^{m} \mu\left(P^{m} h_{n}\right) \leqslant \mu\left(h_{n}\right) \sum_{m \geqslant 0} \alpha^{m}<\infty .
$$

При этом из-за отсутствия в $\widehat{E}$ точки $\Delta$ для любого $x \in \widehat{E}$ найдется номер $n$, при котором $g_{n}(x)>0$, вследствие чего в некоторой окрестности $U$ этой точки $g_{n}>\varepsilon$ при каком-либо $\varepsilon>0$ и $\hat{\mu}(U)<\infty$, что гарантирует внутреннюю регулярность $\hat{\mu}$ [8, гл. 10].

К тому же мера $\hat{\mu}$ субинвариантна для $\widehat{X}$ :

$$
\hat{\mu}(\widehat{P} f)=\mu\left(P\left(f_{E}\right)\right) \leqslant \mu\left(f_{E}\right)=\widehat{\mu}(f), \quad f \in \mathscr{B}_{+}
$$

(cp. (4)). Отмеченные свойства $\hat{\mu}$ позволяют по теореме 4.9 из [10] определить на $(\widehat{E}, \widehat{\mathscr{B}})$ цепь Маркова с переходной вероятностью $q(\cdot, \cdot)$, двойственную цепи $\widehat{X}$ относительно $\hat{\mu}$. 
Воспользовавшись леммой 2 , введем сужение $p^{*}(x, A)=q(x, A), x \in E, A \in \mathscr{B}$, и получим

$$
\int_{A} p(\cdot, C) d \mu=\int_{A} \hat{p}(\cdot, C) d \hat{\mu}=\int_{C} q(\cdot, A) d \hat{\mu}=\int_{C} p^{*}(\cdot, A) d \mu
$$

для $A, C \in \mathscr{B}$, где второе равенство вытекает из определения двойственной цепи. Равенство крайних интегралов в (6) означает двойственность цепи $X$ и цепи с переходной вероятностью $p^{*}(\cdot, \cdot)$.

ЗАмЕчАниЕ 3. В теореме 2 мы ограничились, по-видимому, наиболее важной ситуацией. Однако можно было бы охватить среди некоторых других и случай, когда $E$ оказьвается универсально измеримым в $\widehat{E}$ (по поводу терминологии см. [7], [11]). Именно, потребуем лишь, чтобы мера $\mu$ была $\sigma$-конечна и субинвариантна для $X$ и чтобы в $\widehat{\mathscr{B}}$ нашлось $S \subset E$ с $\mu(E \backslash S)=0$ (разумеется, $S \in \mathscr{B}$ в силу замечания 1 и соотношений $S \in \widehat{\mathscr{B}}$ и $S \subset E)$. Подобно прежнему выясняется, что равенство $\hat{\mu}(A)=\mu(A \cap S)$, $A \in \widehat{\mathscr{B}}$, определяет $\sigma$-конечную меру на $\widehat{\mathscr{B}}$, если функции $h_{n}$ суммируемы по $\mu$, и что в $(\widehat{E}, \widehat{\mathscr{B}})$ определена цепь Маркова с переходной вероятностью $q=q(\cdot, \cdot)$, двойственная $\widehat{X}$ относительно $\hat{\mu}$. Введя переходную вероятность $p^{*}(x, A)=q(x, A \cap S), x \in E$, $A \in \mathscr{B}$, и переписав (6) с новьми $q$ и $p^{*}$ (и заменив во втором и третьем интегралах $A$ на $A \cap S)$, вновь приходим к утверждению теоремы 2 .

5. Расширения неприводимых цепей. В классе неприводимых цепей Маркова удается провести более детальньй анализ связей между различными свойствами исходной цепи $X$ и ее расширения $\widehat{X}$ (по поводу обозначений $\widehat{E}, \widehat{X}$ и т.п. см. конецп. 3 ). С этой целью в этом пункте фиксируется некоторая $\sigma$-конечная мера $\nu$ на $\mathscr{B}$ и постулируется условие

(III) иепь Маркова $X \quad \nu$-неприводима; при этом

а) $\nu$ является максимальной мерой, определяющей неприводимость $(\mathrm{MMOH}), u$

б) все әлементы допустимого набора $H_{0}=\left\{h_{n}\right\}$, определяющего расширения $\bar{X}$ и $\widehat{X}$, минорантны для $X$.

(Разъяснения по поводу терминологии см. в п. 6.) Символом $\hat{\nu}$ будет обозначаться образ $\nu$ при естественном вложении $E$ в $\widehat{E}$.

ЗАмЕчАниЕ 4. Предположения а) и б) из условия (III) мало стеснительны, поскольку, допустив неприводимость $X$, можно, во-первых, как отмечено в п. 6 , найти и ММОН, a, во-вторых, любой допустимьй набор преобразовать в допустимьй же набор минорантных функций (ср. с началом доказательства теоремы 2; при этом прежнее условие $\mu\left(E_{n}\right)<\infty$ заменяется требованием минорантности $\left.E_{n}\right)$.

Мы начнем с изучения связей между минорантными множествами в $E$ и компактными множествами в $\widehat{E}$ (см. теорему 3 ), а затем покажем, что расширение $\widehat{X}$ цепи $X$ наследует некоторые полезные свойства цепи $X$, коль скоро последняя обладает таковьми (см. теоремы 4-6).

Лемма 3. Пусть функиии $s_{1}$ и $s_{2}$ минорантны для $X$. Тогда $G^{\alpha} s_{i} \neq 0, i=1,2$, u $a \leqslant G^{\alpha} s_{1} / G^{\alpha} s_{2} \leqslant b$ в $E$ при некоторых $a, b>0$. Это же утверждение сохраняет силу с $G^{\alpha} s_{i}$ и $E$, замененными на $\overline{G^{\alpha} s_{i}}$ u $\widehat{E}$ соответственно, если функиии $s_{i}$ принадлежат рассматриваемому допустимому набору $H_{0}$. 
Второе утверждение леммы вытекает из первого ввиду отсутствия в $\widehat{E}$ точки $\Delta$. Доказательство первого см. в п. 6.

Лемма 4. Пусть иепь $X$ имеет период $d \geqslant 1$. Хотя бы одно из ииклических для нее множеств (а с ним и любое из них) является минорантным для $X$ тогда $и$ только тогда, когда в $\bar{E}$ нет точки $\Delta$, m.е. когда $\widehat{E}=\bar{E}$.

ДокАЗАТЕЛЬСтво. Пусть $d=1$. Предположим сначала, что $E$ минорантно (в нашем случае $E$ является циклическим множеством по определению). Тогда $p_{m}(\cdot, A) \geqslant$ $\xi(A)$ в $E$ для всех $A \in \mathscr{B}$ при соответствующих $m \in \mathbb{N}$ и мере $\xi \not \equiv 0$, так что $G^{\alpha} 1 \geqslant$ $\alpha^{m} \xi(E)>0$. Но по лемме $3 G^{\alpha} h_{1} \geqslant a G^{\alpha} 1, a>0$, и, следовательно, $\overline{G^{\alpha} h_{1}}>0$, т.е. в $\bar{E}$ нет точки $\Delta$.

Наоборот, пусть известно, что в $\bar{E}$ нет точки $\Delta$. Тогда $\inf \left\{G^{\alpha} h_{1}(x) ; x \in E\right\}>0$. Условие $d=1$ позволяет найти такие $a_{n}>0$ и минорантное множество $B$, что $p_{n}(\cdot, B)$ $>a_{n}, n \geqslant l$, на $B$, где $l \in \mathbb{N}$ (в роли $B$ можно взять любое $C$-множество в смысле [3]). Разумеется, $p_{k}(x, A) \geqslant \eta(A)$ для $x \in B, A \in \mathscr{B}$ при некоторых $k \in \mathbb{N}$ и мере $\eta \not \equiv 0$ на $\mathscr{B}$. Далее, $G^{\alpha} 1_{B}=\sum_{m \geqslant 0} \alpha^{m} P^{m} 1_{B}$ по лемме 3 всюду в $E$ превьшает $b_{1} G^{\alpha} h_{1} \geqslant b_{2}$ (здесь и далее под $b_{i}, i \geqslant 1$, подразумеваются подходящие положительные числа). Равномерная сходимость вьписанного ряда влечет в $E$ оценку

$$
\sum_{m=0}^{N} P^{m} 1_{B} \geqslant b_{3}
$$

с некоторым $N \in \mathbb{N}$. Таким образом, при любом $x \in E$ вьполняется неравенство $p_{m}(x, B) \geqslant b$ при некотором целом $m=m(x)$ из отрезка $[0, N]$, и поэтому всюду в $E$

$$
\begin{aligned}
p_{l+N}(x, B) & \geqslant \int_{B} p_{m}(x, d y) p_{l+N-m}(y, B) \geqslant b_{4} a_{l+N-m} \geqslant b>0, \\
p_{m_{1}}(x, A) & \geqslant \int_{B} p_{l+N}(x, d y) p_{k}(y, A) \geqslant b \eta(A),
\end{aligned}
$$

где $b=b_{4} \min \left\{a_{n} ; l \leqslant n \leqslant N\right\}, m_{1}=l+k+N$, a $A \in \mathscr{B}$ произвольно, что и доказывает минорантность $E$.

Итак, случай $d=1$ рассмотрен, а при $d>1$ надо сослаться на уже доказанное и предложение 2.9 из [2].

В связи с теоремой 3 напомним, что апериодичность цепи $X$ влечет замкнутость семейства минорантных множеств относительно конечных объединений [2, следствие 2.1].

Теорема 3. Каков бы ни был компакт $K \subset \widehat{E}$, множество $K \cap E$ покрывается обвединением конечного числа множеств, минорантных для $X$. Напротив, каждое минорантное множество содержится в некотором компакте $K \subset \widehat{E}$.

ДокАЗАТЕльство. Лемма 4 делает теорему очевидной, если в $\bar{E}$ нет точки $\Delta$, и этот случай далее исключается. Кроме того, мы вправе считать $d=1$ (ср. конец предыдущего доказательства).

Снова выделим элемент $h_{1}$ из рассматриваемого допустимого набора и заметим, что $g \equiv \overline{G^{\alpha} h_{1}} \neq 0$ в $\widehat{E}$ в соответствии с леммой 3 . Пусть $K-$ некоторый компакт в $\widehat{E}$. Расширив его при необходимости, будем считать $\hat{\nu}(K)>0$ (определение $\hat{\nu}$ см. в начале 
пункта). Из непрерьвности $g$ в $\widehat{E}$ вьводится неравенство $g>a$ на $K$, а тем самым и на $K \cap E$ при некотором $a>0$. Следовательно, $\sum_{n=1}^{N} P^{n} h_{1}$ превьшает на $K \cap E$ некоторое положительное число, если $N \in \mathbb{N}$ достаточно велико (ср. с выбором $N$ в предыдущем доказательстве). Отсюда с учетом соотношений $\hat{\nu}(K)=\nu(K \cap E)>0$ вытекает минорантность множества $K \cap E$ (см. [2, предложение 2.11]), и первое утверждение теоремы доказано.

Фиксируем теперь минорантное $A \subset E$ и заметим, что оно содержится в $\left\{x: G^{\alpha} 1_{A}(x)\right.$ $>1 / 2\} \subset\{x: g(x)>b\}$, где $b>0$ достаточно мало и вновь использована лемма 3 . Множество $K=\{x \in \widehat{E}: g(x) \geqslant b\}$ компактно в $\widehat{E}$, поскольку $g(x) \rightarrow 0$ при $x \rightarrow \Delta$ в топологии пространства $\bar{E}$, и оно, очевидно, содержит $A$.

Теорема 4. Расширение $\widehat{X} \hat{\nu}$-неприводимо. Более того, $\hat{\nu}$ является ММОН для $\widehat{X}$, а параметры сходимости $X$ и $\widehat{X}$ совпадают друг с другом.

ДокАЗАтЕльство. Определим $g$ так же, как и в доказательстве теоремы 3. Задав $A \in \widehat{\mathscr{B}}$ с $\hat{\nu}(A)>0$, подберем компактное в $\widehat{E}$ множество $K \subset A$ с $\hat{\nu}(K)>0$. В $K_{1}=K \cap E$ содержится минорантное подмножество (см. п. 6), и применив лемму 3 , получаем в $E$ неравенство $G^{\alpha} 1_{K_{1}} \geqslant a g$ с некоторым $a>0$. Пусть $f_{n} \in C\left(\bar{E}_{n}\right)$, причем $1 \geqslant f_{n} \geqslant 1_{K}$ и $f_{n} \rightarrow 1_{K}$ при $n \rightarrow \infty$. Из неравенства $G^{\alpha}\left[\left(f_{n}\right)_{E}\right] \geqslant a g_{E}$ вьводим неравенство $\overline{G^{\alpha}} f_{n} \geqslant a g, n \geqslant 1$, а затем, переходя к пределу, и неравенство $\overline{G^{\alpha}} 1_{K} \geqslant a g$ (здесь $\overline{G^{\alpha}}$ - аналог $G^{\alpha}$ для $\bar{X}$ ). Тем более, $\bar{G}^{\alpha} 1_{A}>0$ в $\widehat{E}$ и $\hat{\nu}$-неприводимость цепи $\widehat{X}$ установлена.

Чтобы объяснить, почему $\hat{\nu}$ служит ММОН для $\widehat{X}$, нужно для $A \in \widehat{\mathscr{B}}$ с $\hat{\nu}(A)=0$ доказать любоеиз равносильных равенств $\hat{\nu}\left(A_{1}\right)=0$ или $\nu\left(A_{1} \cap E\right)=0$, где $A_{1}=\{x \in \widehat{E}$ : $\left.\widehat{P} \widehat{G} 1_{A}>0\right\}$. Однако второе из них очевидно, так как $A_{1} \cap E=\left\{x \in E: P G 1_{A \cap E}>0\right\}$ и $\nu$ служит ММОН для $X$.

Остается удостовериться в осуществлении неравенств

$$
R \geqslant \widehat{R} \quad \text { и } \quad \widehat{R} \geqslant R,
$$

где $R$ (соответственно $\widehat{R}$ ) - параметр сходимости цепи $X$ (цепи $\widehat{X}$ ). Рассмотрим $A \in \widehat{\mathscr{B}}$ с $\hat{\nu}(A)>0$ и положим $S_{A}=\sum_{n \geqslant 0} r^{n} \widehat{P}^{n} 1_{A}$, где $r>R$ произвольно. Всюду в $E$ согласно условию (III) и определению параметра $R$ (см. п. 6$)$ имеем

$$
S_{A}=\sum_{n \geqslant 0} r^{n} P^{n} 1_{A \cap E}=\infty
$$

поскольку $\nu(A \cap E)>0$. Иными словами, $E \subset B=\left\{x \in \widehat{E}: S_{A}(x)=\infty\right\}$ и потому $\hat{\nu}(\widehat{E} \backslash B)=0$. Итак, $S_{A}=\infty \hat{\nu}$-почти всюду, т.е. $r \geqslant \widehat{R}$, что исключает случай $\widehat{R}>R$ и доказывает первое из неравенств $(7)$.

Наоборот, пусть $r>\widehat{R}$, и пусть $A \in \mathscr{B}$ и $\nu(A)>0$. По замечанию 1 найдется $B \in \widehat{\mathscr{B}}$, для которого $B \cap E=A$, причем $S_{B} \equiv \infty$ в $\widehat{E}$ ввиду равенства $\hat{\nu}(B)=\nu(A)$ и определения $\widehat{R}$. Отсюда и $S_{A} \equiv \infty$ в $E$, так как $P^{n} 1_{A}=\widehat{P}^{n} 1_{B}$ в $E$. Остается по аналогии с предыдущим вывести второе из неравенств в (7).

ТЕОрема 5. Если цепь $X$ возвратна, то и $\widehat{X}$ возвратна. 
ДоказАтельство. Пусть $f \in \widehat{\mathscr{B}}_{+}$и $\hat{\nu}(f)>0$. Рассмотрим множество $A=\{x \in \widehat{E}$ : $\widehat{G} f(x)=\infty\}$ и его дополнение $B=\widehat{E} \backslash A$. Ввиду возвратности исходной цепи $\widehat{G} f=$ $G\left(f_{E}\right)=\infty$ на $E$, так как $\nu\left(f_{E}\right)=\hat{\nu}(f)>0$. Таким образом, $A \supset E$ и $\hat{\nu}(B)=$ $\nu(B \cap E)=0$. Последнее было бы невозможно в случае непустого $B$, поскольку в этом случае субинвариантность $\widehat{G} f$ влекла бы неравенство $\hat{\nu}(B)>0$ (см. [2, предложение 5.1]). Итак, $B$ пусто для всех $f$ указанного вида.

В связи со следующей теоремой напомним, что возвратность неразложимой цепи Маркова влечет ее лиувиллевость (см. п. 6). Легко также проверить, что если все меры вида $p(x, \cdot)$ абсолютно непрерьвны относительно $\nu$, то свойства лиувиллевости и $\nu$-лиувиллевости равносильны.

ТЕОрема 6. Если иепь $X$ является $\nu$-лиувиллевой, то $\widehat{X}$ является $\hat{\nu}$-лиувиллевой.

ДокАЗАТЕльство. Пусть $\sigma$-конечная мера $\mu \not \equiv 0$ инвариантна для $X$ и абсолютно непрерьвна относительно $\nu$, и пусть $W$ - совокупность всех мер на $\widehat{\mathscr{B}}$, обладающих такими же свойствами по отношению к $\widehat{X}$ и $\hat{\nu}$. Возврашаясь ко второму абзацу доказательства теоремы 2 , заключаем, что $\hat{\mu} \in W$, где $\hat{\mu}$ - образ $\mu$ при естественном вложении $E$ в $\widehat{E}$. Далее, каждая мера $\eta \in W$ эквивалентна $\hat{\nu}$ (ведь равенства $\eta(A)=\eta\left(\widehat{P} 1_{A}\right)=0$ с $A \in \widehat{\mathscr{B}}$ влекут существование $x \in \widehat{E}$, при котором $\hat{p}(x, A)=0$, а тем самьм по теореме 4 и равенство $\hat{\nu}(A)=0$ ). Таким образом, всякая мера $\eta \in W$ эквивалентна $\hat{\mu}$ и обладает производной Радона-Никодима $h=d \eta / d \mu$. Для $f \in \widehat{\mathscr{B}}_{+}$получаем

$$
\eta(f)=\eta(P f)=\hat{\mu}(h \widehat{P} f)=\mu\left(h_{E} P\left(f_{E}\right)\right)
$$

и $\eta(f)=\hat{\mu}(h f)=\mu\left(h_{E} f_{E}\right)$. Отсюда следует инвариантность для $X$ меры $\mu_{1}(A)=$ $\mu\left(h_{E} 1_{A}\right), A \in \mathscr{B}$, а стало быть, $\mu_{1}=k \mu$ при некотором постоянном $k \geqslant 0$. Это означает, что $h_{E}=k \mu$-почти всюду и в силу последних цепочек равенств $\eta=k \hat{\mu}$.

6. Добавление. В этом пункте собраны некоторые сведения о неприводимых цепях Маркова.

Пусть на $\sigma$-алгебре $\mathscr{B}$ задана $\sigma$-конечная мера $\nu$. Если исходная цепь $X \nu$-неприводима и $G 1_{A}=0 \nu$-почти всюду всякий раз, когда $\nu(A)=0$, то $\nu$ назьвают максимальной мерой, определяющей неприводимость (ММОН). Требование неприводимости $X$ относительно некоторой меры равносильно существованию $\mathrm{MMOH}[2]$, [3], и поэтому впредь упомянутую меру $\nu$ будем считать ММОН для $X$ (ср. с условием (III)).

Функция $s: E \rightarrow[0, \infty)$ назьвается минорантной для $X$ (соответствующий английский термин - a small function), если $\nu(s)>0$ и существуют $b>0$, натуральное $m$ и мера $\xi$ на $\mathscr{B}$ такие, что $\xi(E)>0$ и $p_{m}(x, A) \geqslant b s(x) \xi(A)$ для всех $x \in E, A \in \mathscr{B}[2]$. В этом же случае и мера $\xi$ именуется минорантной. Множество $A \in \mathscr{B}$ по определению минорантно, если его индикатор минорантен.

Для любой функции $f \in \mathscr{B}+\mathrm{c} \nu(f)>0$ и любой минорантной функции $s$ существуют $b>0$ и $m \in \mathbb{N}$, при которых

$$
P^{m} f \geqslant b s
$$

(см. [2, предложение 2.7]), что вместе с неравенством $G^{\alpha} f \geqslant \alpha^{m} G^{\alpha} P^{m} f$ влечет неравенство $G^{\alpha} f \geqslant a G^{\alpha} s$ с некоторым $a>0$. Более того, отсюда следует первое утверждение леммы 3 из п. 5 . 
Для цепи $X$ можно указать параметр сходимости $R$, т.е. такое число $R>0$, что

a) $G^{\alpha} s<\infty \nu$-почти всюду, если $0 \leqslant \alpha<R$, а функция $s$ минорантна, и

б) $G^{\alpha} f \equiv \infty$, если $\alpha>R$, a $f \in \mathscr{B}_{+}$и $\nu(f)>0$

(в отличие от предыдущего изложения здесь определение (1) используется при любом $\alpha \geqslant 0)$. Если $\sigma$-конечная мера $\mu \not \equiv 0$, заданная на $\mathscr{B}$, субинвариантна, то $0<\mu(s)<\infty$ для любой минорантной функции $s$. Действительно, неравенства $\mu(f) \geqslant \mu\left(P^{m} f\right) \geqslant b \mu(s)$, в которых функция $f$ указанного вида произвольна, а $m$ и $b$ подобраны в соответствии c $(8)$, исключают случай $\mu(s)=\infty$. Выбрав же минорантную функцию $f$ с $\mu(f)>0$ (см. [2, предложение $2.11(\mathrm{iv})])$ и сославшись на лемму 3 , получим $\mu\left(G^{\alpha} s\right) \geqslant c \mu\left(G^{\alpha} f\right)$ при некотором $c>0$, откуда $\mu(s)>0$.

Возвратная иепь $X$ является лиувиллевой (это хорошо известно для цепей, возвратных по Харрису [3]). В самом деле, если $s$ минорантна, то в силу только что сказанного для любой из $\sigma$-конечных мер, инвариантных для $X$, можно указать $k>0$, при котором $k \mu(s)=1$, откуда вытекает пропорциональность $\mu$ и меры $\pi_{s}$, фигурирующей в теореме $5.2[2]$.

В заключение напомним [2], что период цепи $X$ определяется как наибольшее из натуральных $d$, при которых найдутся такие попарно непересекающиеся непустые множества $E_{i} \in \mathscr{B}, 0 \leqslant i \leqslant d-1$ (называемые ииклическими множсествами), что $p\left(x, E \backslash E_{j}\right)=0$ при $x \in E_{i}$ и $j \neq i+1(\bmod d)$. Цеп $X$ апериодична, если $d=1$.

\section{СПИСОК ЦИТИРОВАННОЙ ЛИТЕРАТУРЫ}

[1] Шур М. Г. Инвариантные меры для цепей Маркова и феллеровские расшшрения цепей // Теория вероятн. и ее применения. 1981. Т. 26. № 3. С. 496-509.

[2] Нуммелин Э. Общие неприводимые цепи Маркова и неотрицательные операторы. М.: Мир, 1989.

[3] Orey S. Lecture Notes on Limit Theorems for Markov Chain Transition Probabilities. London: Van Nostrand, 1971.

[4] Ж данок А. И. Асимптотическое поведение цепей Маркова и инвариантные конечно-аддитивные меры // Дисс. ... к. ф. -м. н.. Рига: Латв. гос. ун-т 1981.

[5] Knight F. Note on regularization of Markov processes // Illinois J. Math. 1965. V. 9. №3. P. 548-552.

[6] Люмис Л. Введение в абстрактный гармонический анализ. М.: ИЛ, 1956.

[7] Dellacherie C., Meyer P. A. Probabilités et potential (chapitres I-IV). Paris: Hermann, 1975.

[8] Халмош П. Теория меры. М.: ИЛ, 1953.

[9] Кемени Дж., Снелл Дж., Кнепп А. Счетные цепи Маркова. М.: Наука, 1987.

[10] Ревюз Д. Цепи Маркова. М.: РФФИ, 1997.

[11] Куратовский К. Топология.. Т. 1. М.: Мир, 1966. 